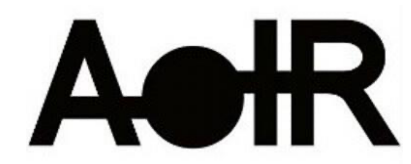

Selected Papers of \#AoIR2021:

The 22nd Annual Conference of the Association of Internet Researchers Virtual Event / 13-16 Oct 2021

\title{
COLLABORATING AT MICROSCOPIC AND \\ MASSIVE SCALES: THE CHALLENGE AND VALUE OF COVID ISOLATION FOR CRITICAL INTERNET STUDIES
}

\author{
Andrew Herman \\ Wilfred Laurier University \\ Mary Elizabeth Luka \\ University of Toronto \\ Annette Markham, \\ RMIT \\ Danielle Dilkes \\ University of Toronto \\ Riccardo Pronzato \\ ILUM University \\ Devina Sarwatay \\ University of Hyderabad \\ Rebecca Carlson \\ Tokyo University \\ Fiona Stirling \\ Abertay University
}

\section{Keywords}

Covid, sensemaking, digital collaboration, community, digital methodologies

\section{Short Abstract (Word count: 247)}

Herman, A., Luka, M.E., Markham, A., Dilkes, D., Pronzato, R., Sarawatay, Carlson, R., Stirling, R., Stirling, F. (2021, October) Collaborating At Microscopic And Massive Scales: The Challenge And Value Of Covid Isolation For Critical Internet Studies. Paper (or panel) presented at AolR 2021: The 22nd Annual Conference of the Association of Internet Researchers. Virtual Event: AolR. Retrieved from http://spir.aoir.org. 
Global events like a pandemic or climate change are massive in scope but experienced at the local, lived, microscopic level. What sorts of methodologies and mindsets can help critical internet researchers, functioning as interventionists or activists, find traction by oscillating between these levels? How can we push (further) against the boundaries of research methods to build stronger coalitions and more impactful outcomes for social change among groups of scholars/researchers? This panel presents four papers addressing these questions based on a large scale online autoethnography in 2020. This "Massive/Micro" project simultaneously used and studied the angst and novelty of isolation during a pandemic, activating researchers, activists, and artists to explore the massive yet microscopic properties of COVID-19 as a "glocal" phenomenon. The challenge? Working independently and microscopically through intense focus on the Self but also working with distributed, largely unknown collaborators, in multiple platforms. The emerging shape of the project itself showcases the challenges and possibilities of how research projects at scale can (or don't) reflect and build social movements. The panel's four papers situate the project through a kaleidoscope of perspectives featuring participants from 7 countries, who variously explore: the value of the project for precarious or early career researchers, how MMS worked as both collaborative space and critical pedagogy, how non-institutional or playful experimentation in asynchronous collaborations can lead to new synergies; and how MMS developed an independent life of its own, beyond studying COVID to generating multiple communities of future digital research practice.

\section{Extended description of the panel: 600-800 words (word count 785 )}

Global events like a pandemic or climate change are massive in scope but experienced at the local, lived, microscopic level. What sorts of methodologies and mindsets can help critical internet researchers, functioning as interventionists or activists, find traction by oscillating between these levels? How can we push (further) against the boundaries of research methods, disciplines, and institutional boundaries to build more independent coalitions and more impactful outcomes for social change among groups of scholars/researchers? This panel presents four papers addressing these questions based on the experiences of engaging in a large scale online autoethnography in 2020.

The project was initially cast as a call for papers focused on the question: How is COVID 19 helping us think about socio-technical systems of humans, machines and the planet at different registers and dynamics of scale? With poignant contributions from more than 150 artists, researchers, and activists from 26 countries, the the "Massive and Microscopic Sensemaking in times of COVID 19" project (hereafter MMS) project grew into a large-scale experiment to facilitate collaborative yet distributed research, using various digital platforms (cf: Markham, Harris and Luka, 2020). The aim of MMS was to simultaneously use and study the angst and novelty of isolation during COVID-19 lockdowns, to activate researchers, activists, and artists to explore, through collaborative autoethnographic approaches, the massive yet microscopic properties of COVID-19 as a "glocal" social phenomenon (Roudometof, 2016).

The challenge? Working independently and microscopically through intense focus on the Self as research subject, but also engaging virtually with largely unknown 
collaborators on asynchronous digital platforms. The emerging shape of the project itself showcases the challenges and possibilities of how research projects at scale can (or cannot) reflect and build social movements, how serendipity plays a strong role in emergent research design, how independence and interdependence are emergent and ad hoc temporal qualities of large scale collaborations rather than built-in or top-down properties.

This was a unique situation. The size of the group was larger than could be managed as a whole. Individuals were situated in almost all the world's timezones. Each person had varying degrees of ability to participate on a regular basis. Finally, and perhaps most importantly, the ongoing stress and anxiety around COVID-19 meant this was a vulnerable group. We needed to find a way for people to collaborate without feeling pressured to be online all the time, respond to others, or respond in a particular time frame.

Facebook, despite the facilitators' misgivings around the politics of this platform, became the primary mode of digital communication and collaboration between participants in the project. The socio-technical affordances of the platform had a noticeable effect on the interactions and sociality of the group. A rhythm of collaborative community emerged, as posts and responses to prompts influenced other posts and responses. People also indicated they felt encouraged by others' posts, and some remarked that this enabled them to reply when otherwise, they might have been reserved for some reason, whether by insecurity about their own thoughts or shyness. Whether this was because they could see how happy people were when others responded positively to their posts, even if that was only in the form of a like, love, laugh, or care icon. Although a close level analysis of the Facebook group has not yet been conducted yet, anecdotal evidence indicates that the experience was unfolding more like a focus group than an individual autoethnographic immersion.

Over a year later, the project is ongoing and thus far, has generated more than 50 papers and 15 audio/visual exhibition performances. In the Panel, several participants from MMS reflect on how this collaboration worked, what it meant, and what it suggests for future research practice. The papers situate the project through a kaleidoscope of perspectives illustrating how MMS developed an independent life of its own, beyond studying COVID to generating multiple communities of future digital research practice.

1. Paper 1 uses MMS as an example of an independent, non-institutionalized project to discuss the contradictory and precarious nature of online collaborations as well as their potential for positive change;

2. Paper 2 discusses theoretical implications emerging from their auditory sensemaking during MMS, and the value of 'oscillating' between micro- and macro-focused methodologies;

3. Paper 3 focuses on MMS as a critical pedagogy for early career participants and how this impacted their researcher identities;

4. Paper 4 circles back to the critical and ethical value of the project overall by 
focusing initially on the MMS videos, then moving towards a manifesto for large scale collaborations.

\section{References}

Markham, A. A. Harris, and M. E. Luka. (2020). "Massive and Microscopic Sensemaking During COVID-19 Times". Qualitative Inquiry 0 10.1177/1077800420962477.

Roudometof, V. (2016) "Theorizing glocalization: Three interpretations". European Journal of Social Theory Vol. 19(3): 391-408 


\title{
REMOTELY REWILDING: PRECARIOUSLY COLLABORATING DURING THE COVID PANDEMIC (1145 words)
}

\author{
Rebecca Carlson \\ Tokyo University \\ Fiona Stirling \\ Abertay University

\section{Background}

In this paper, the authors, based in the UK and Japan, reflect on the possibilities and barriers for digitally mediated forms of emergent research collaborations using autoethnographic methods. Both authors participated in the "Massive and Microscopic Sensemaking in times of COVID 19 project" (hereafter MMS), an independent, large-scale online social community collaboration that incorporated over 100 scholars from various academic backgrounds, from 26 countries. Led by Annette Markham, Anne Harris and M.E. Luka in early 2020, the project was a direct response to the COVID-19 pandemic. It utilised social media and a 21-day series of asynchronous, self-guided activities to bring together academics, artists, and activists for collaborative research around the everyday impact of isolation and lockdowns. Participants practiced critical autoethnographic methods independently (working mostly alone), and also interdependently (alongside each other), completing, sharing, and commenting on their and others' reflective writing exercises. The authors met through this event, finding themselves methodologically interconnected through the project process. Using their shared experience of the MMS project as a point of focus, in this paper, the authors discuss the precarious and contradictory nature of online research collaborations along with their restorative potential for broader academic landscapes.

Although more recent internet-mediated collaborations may be a response to living in various frames of relative isolation over the past year, they more broadly reflect the negotiation of modern experiences of social isolation that predate the pandemic. Digital collaborations embody both the affordances and the limitations of their communicative forms, simultaneously potentializing connection and dis-connecton, atomization and collectivization. In addition, such collaborations reflect the modern nature of intellectual labor, where researchers must take on independent responsibility for their productivity, but remain subject to limiting academic standards. What then does academic 'work' look like now? Feel like? Exactly what activities and outputs are recognized as productivity right now?

\section{Shifting spaces and emerging questions}

In the wake of a destabilizing pandemic, an unprecedented number of online research collaborations have flourished, at times with little direct institutional support or guidance. Both authors joined multiple digital collaborations, in addition to MMS, that often formed relatively organically, drawing on resources and initial connections already at hand (well-established mailing lists for example). At the same time, both also experienced, or 
witnessed, the ways that academic requirements prioritized researchers to work independently, and in ways that would lead to standardized research outputs such as first-author journal publications. These two conditions can make online and large-scale experimental projects relatively unsustainable in the long term. McCulloch (2017) advises that such institutional processes put pressure on how and what academics write, which shape career goals, disciplinary values, and scholarly identity. The roles we might choose to take on then, or the energy we might choose to commit, may diminish in value and importance if collaborative opportunities do not fit accepted institutional modes of productivity. Ultimately, we then also fail to take on new voices and ideas, and to innovate in our research methods, analysis and publication styles. To produce research that accurately reflects modern experiences, the authors argue that it is necessary to find ways for online collaborations to form in playful, thoughtful, digital sandboxes, and to have such practices and their diverse outcomes be valued in the academy.

\section{A Window into Precarities and Possibilities}

Nordback et al. (2021) suggest that while academic identity appears to be 'placeless' it is in fact influenced by time and place. COVID-19 displaced all academics, shifting them from known physical spaces to remote digital unknowns. The navigation of these new boundaries and norms can be jarring and exhausting; this materialized for author 1 who had not previously held a professional 'online' identity and for author 2, in the loss of her in-person field site and the participatory side of her ethnographic work. Yet, both authors also felt pulled together with other researchers in new and exciting ways. Such novel engagement can prompt (re)consideration of long held attitudes and approaches to activities like collaboration. Hernaus et al. (2018) identify a general tension in academia between the presumption of a generous spirit of collaboration, and the competitive pressure to perform and outperform others. This can encourage 'knowledge-hiding', where information and ideas are intentionally concealed. When exposed to the openness of the digital landscape, where comments are committed to permanent record and thoughts are transcribed onto pages that will likely exist beyond single lifetimes, the process of producing knowledge is transformed from one which is insular and driven by the need for immediate production of evidence, to an ongoing, never-ending assemblage process rewarded by the potential for meaningful interconnection.

Ethnographic research is humanistic. Its purpose is precisely to reach across lived experiences, beginning in the everyday subjectivity of the researcher, to something collectively meaningful. Forging collaborations with other researchers helps us shift these registers. Methods such as autoethnography, in which the researcher becomes the subject of the research with the purpose of examining an aspect of the lived experience in cultural contexts (Ellis, 2004), can provide a direct and immediate lens of reflexivity for 'thick description' to emerge (Geertz, 1973). Considering, and facilitating, online research in this way emphasizes the interconnected experience central to digital mega-collaborating and illustrates the challenges, and possibilities, present in attempting to represent the co-production of knowledge generated through such projects. Rinehart and Earl (2016) posit that such methodology is also an inherently caring practice, thereby resisting some of the pressure inducing practices of the 
neoliberal academy.

Moriarty (2019) develops the concept of 'rewilding' as an apt metaphor for renewing the diverse landscape of Higher Education via replenishing new approaches to pedagogy and research. 'Neoliberalism has choked too much of what was nourishing about Higher Education,' she warns 'and yet, the answer is not to return to our past'. MMS experimentally pointed us to a possible future, one of online collaborations informed by autoethnographic methods, a weaving of the microscopic self into the massively social, rich with rewilding promise.

\section{References}

Blalock, A. E., \& Akehi, M. (2018). Collaborative autoethnography as a pathway for transformative learning. Journal of Transformative Education, 16(2), 89-107.

Ellis, C. (2004). The ethnographic I: A methodological novel about autoethnography. Altamira.

Geertz, C. (1973). The Interpretation of Cultures. Basic books.

Hernaus, T., Cerne, M., Connelly, C., Vokic, N. P., \& Škerlavaj, M. (2019). Evasive knowledge hiding in academia: when competitive individuals are asked to collaborate. Journal of Knowledge Management.

McCulloch, S. (2017, February 9th) The importance of being REF-able: Academicwriting under pressure from a culture of counting. Impact Blog, London School of Economics.https://blogs.Ise.ac.uk/impactofsocialsciences/2017/02/09/theimportance-of -being-ref-able-academic-writing-under-pressure-from-a-culture-ofcounting/

Moriarty, J. (Ed.). (2019). Autoethnographies from the Neoliberal Academy: Rewilding, Writing and Resistance in Higher Education. Routledge.

Nordbäck, E., Hakonen, M., \& Tienari, J. (2021). Academic identities and sense ofplace: A collaborative autoethnography in the neoliberal university. Management Learning, 13505076211006543.

Rinehart, R. E., \& Earl, K. (2016). Auto-, duo-and collaborative-ethnographies: "Caring" in an audit culture climate. Qualitative Research Journal. 


\section{THE SOUND OF CRITICAL SENSEMAKING: REVISITING ORALITY, SOUND \& VOICING DURING THE ISOLATION OF GLOBALTRAUMA}

Andrew Herman

Wilfrid Laurier University

Annette Markham

RMIT University

\section{Background}

What is the "sound" of critical sense-making? This paper draws on the experiences of the authors to revisit--through sound and aurality--the methodological mandate of the "Massive and Microscopic Sensemaking in times of COVID 19" collaborative autoethnography project (hereafter MMS) explored by this panel. MMS was initially cast into sense by Annette Markham and Anne Harris in the spring of 2020 as most of world was thrown into various degrees of lock down in order to prevent the spread of COVID 19 (Markham and Harris, 2020; Markham, Harris and Luka, 2020) to pose a basic question:How is COVID 19 helping us think about socio-technical systems of humans, machines and the planet at different registers and dynamics of scale, or how do intimate occurrences with microscopic properties connect with a planetary-scale event with potentially massive outcomes? (Markham, Harris \& d Luka, 2020: 1) The ongoing project has grown to involve more than 150 researchers and artists from 26 countries.

\section{From ocular lens to aural ear: A lingering challenge in social research}

A multiplicity of diverse modes of scholarly and art-based autoethnographic practices have been deployed as critical sense-making on the MMS project, largely story-telling via prose and poetry or visual artistic practices of image making. In this paper, the collaborating authors shift attention from the "autoethnographic lens" of visuality towardsthe "autoethnographic ear" of sound and aurality. We explore some of the lessons of theMMS project for digital methodologies that foreground the acoustic space of audition and aurality. The concept of acoustic space is drawn from the work of Canadian communication theorist Marshall McLuhan (1962) and comprador intellectual spirit in medium theory, Walter Ong (1982). For both, the story-telling practices of oral cultures privilege a collaborative and communal sonorous way of knowing and being, phenomenologically embodied in ways that visual signs of writing and image are not, enveloping the speaker and listener in a shared space of audition (Cavell, 2003).

What are the practical accomplishments of a performative grounded theory centrally figured around the aurality and acoustic? One way of hearing and listening our way across these articulations entails working with and through the concept and phenomenology of "resonance". For McLuhan, "resonance" is a way of thinking 
about the relation of sounds in acoustic space and the way they converge in degrees of consonance and dissonance (c.f. Young, 530). Acoustic space, as Cavell points out, isnot "a physical space in the sense of a container; in the acoustic field, it is the sound itself that creates the space" (Cavell, 203: 157). The sonic convergences of resonancealso invite an understanding of acoustic space as Heideggerian ethos, an abode or place of dwelling from within which one can engage the world.

\section{Critically analyzing how we make sense of COVID: Acoustic punctums andvibratory experiences}

The story that unfolds in this presentation starts from two very different such abodes. The starting point of Markham's story is a stretch of beach in Northern Europe where sea meets sand along a wind-swept beach (cf. Markham, 2020). As the author walks along the beach during the early COVID-related lockdowns of 2020, she repeats the ancient Kundalini mantra of Sat Nam. She sings it, whispers it, screams it, swallows it. The repetition of the mantra is key to the author's grounding of the self in the midst of a larger world that has been dislocated by COVID 19. In Pettman's words, the invocation of the mantra is an "acoustic punctum", a sonic pinprick in the fabric and flow of the quotidian that organizes an aural ethos from which she attempts to make sense of the pandemic (Pettman, 2017: 5). The Sanskrit mantras also give notice to the plasticity of the self as embodied subject in aural space, "as Sanskrit as a language was supposedlydesigned to make changes in our bodies as they entered our ears, or as we shape themin our mouths" (Markham, 2020: 3). This continual chanting also becomes Author \#1's central focus for analyzing patterns, both in the physical environment and the digital world of news about and around COVID-19 through grounded theory. The resonant features of the aural are reflected in later writing and image making that reflect the repetition of the mantra, or distinguish the whispering from screaming, through long paragraphs without any punctuation or staccato-punctuated one word paragraphs, as if shouting.

At the same time that Markham is screaming Sanskrit to the sky against the wind in Denmark, Herman is broadcasting from his basement in Toronto, using sonic intimacy to break the sensory loneliness of isolation. These are guttural outcries-- viscerally felt entreaties connecting the microscopic resonances in and of the body to the massive and incomprehensible weight and power of a pandemic to a public-in -ormation. Lookingback at the playlists, Herman recognizes a pandemic-driven compulsion to be engulfed by music on the low-end frequencies of the bass. As Henriques' (2011) analysis of the affective power of low-end sonority of reggae sounds systems suggests, these soundscapes can foster spaces of empowerment, resistance, and community. As the weeks pass, Herman playfully experiments with song and spoken word to generate "lively" acoustic resonance between the Self and the imagined world of listeners, hopefully conjuring: "vibratory encounters [that] work to unravel selfcertainty and recastour sensed surroundings, making us rethink what we can do how we operate" (Jasen, 2016: 3). Each broadcast is a form of sensemaking to make prefigurative sense of the altered, damaged and destroyed rhythms of everyday life 
under the conditions of COVID-19.

From these points of origin-a beach in Europe and a basement in Canada- the authorswork their ways towards one another across different socio-technical registers of the massive and micro through the acoustic space of collaborative story-telling and performative grounded theory. Drawing from Jasen's (2016) work on the materiality of sonic experience, we find that in our listening to each other, the "sonic body" of the pandemic is microscopic in quality, as sonorous sensemaking passes intercorporeally, to generate affective harmonic frequencies of resonance in that 'moved us' into a massive shared sensory space in those experiential moments of becoming otherwise, before COVID-19 yielded fits full meanings. What does this quality of inquiry bring to the study of global crisis, to sonically illuminate the different registers of the scale between the "micro" and the "massive" as it thrums along each node of their articulations?

\section{References}

Cavell, R. (2003). McLuhan in Space. Toronto: University of Toronto

PressHenriques, J. (2011) Sonic Bodies. New York: Bloomsbury.

Jasen. P. (2016). Low End Theory: Bass, Bodies and the Materiality of SonicExperience. New York: Bloomsbury.

Markham, A. (2020) "Pattern Recognition: Using Rocks, Wind, Water, Anxiety, and Doom Scrolling in a Slow Apocalypse (to Learn More About Methods for Changing theWorld)". Qualitative Inquiry 0 10.1177/1077800420960191

Markham, A. and A. Harris. (2020). "Prompts for Making Sense of A Pandemic". Qualitative Inquiry 0 10.1177/1077800420962487

Markham, A., A. Harris, and M. E. Luka. (2020). "Massive and MicroscopicSensemaking During COVID-19 Times". Qualitative Inquiry 0 10.1177/1077800420962477.

McLuhan, M. (1962). The Gutenberg Galaxy. Toronto: University of Toronto Press

Ong, W. (1982). Orality and Literacy. New York: Methuen.

Pettman, D. Sonic Intimacy. (2017). Palo Alto: Stanford University Press.

Young, L. C. (2019) "The Innis-McLuhan Field: In Search of Media Theory". CanadianJournal of Communication Vol. 44: 527-544. 


\title{
AUTOETHNOGRAPHY AS CRITICAL PEDAGOGICAL PRACTICE
}

\author{
Danielle Dilkes \\ Ontario Institute for Studies in Education, University of Toronto \\ Riccardo Pronzato \\ IULM University \\ Devina Sarwatay \\ University of Hyderabad
}

\section{Background}

The Massive-Micro Sensemaking (MMS) project drew on critical autoethnographic methodologies to engage multiple participants in simultaneously independent and collaborative sense-making during the COVID-19 pandemic. The 21-day series of prompts, distributed on Facebook and a mailing list to a large group of participants, prompted individual level self-reflection and exploration while creating a sense of globalconnectedness. Each participant experienced the project in a unique way, and in this paper, we elaborate how this project was not simply a study of COVID-19, but an eventthat brought together unknown researchers at all different levels. The authors of this paper, PhD students based in Canada, India and Italy, bring their early career perspective on the epistemological value of this form of collaboration. We offer three distinctive examples of how this project positively challenged common assumptions about how inquiry might operate outside institutional and disciplinary boundaries, and how communities of practice develop; about how the techniques used for building reflection among participants of MMS could be adapted to our own pedagogical work; and how the same patterns in the global MMS project could be witnessed in smaller clusters of learners. These examples illustrate how MMS affected our own pedagogicalpractices, identities as scholars, and our ideas about how inquiry in this era might operate.

\section{Exploring the Nexus of Identity through Autoethnography}

For many people, COVID-19 stay-at-home orders disrupted daily routines and relocatedprofessional, academic, social, familial, and other daily practices to the same physical and virtual spaces. This blurred the boundaries between previously distinct identities. Asa student, academic, educational developer, daughter, sister, wife, I (Dani) felt this blurring acutely. This sudden unboundedness forced me to critically reconsider my own identity and practices while simultaneously offering a release from the confines of pre- existing boundaries of practice. As Wenger notes, reconciling multiple selves "into a way of being in the world" can be one of the largest challenges faced by individuals (1999, p. 160). 
The MMS project provided a means for me to critically reflect on the nexus of these identities, demonstrating how autoethnography can perform as an intersectional praxis allowing individuals to reflect on how they are "always both particular and plural at the same time" (Alexander, 2021, p. 32) while understandingthemselves and others as situated in larger macro-communities. In this way, critical autoethnography is liberating, as it allows participants to engage in a form of communityrule-breaking by practicing outside of traditional boundaries, thus empowering them to critically reflect on, and potentially subvert, existing bounded practices and the systems in which they occur. In the context of higher education, autoethnography has the potential to transform professional identities, collaborative pedagogical practices, and the communities where both are situated.

\section{Adapting the 21-Day Autoethnography Challenge as a research tool}

I (Riccardo) applied the MMS approach in my doctoral research to investigate the reception of algorithmic media by individuals in everyday life. In this field, users are often depicted as oppressed subjects, unable to protect themselves from opaque, discriminatory and exploitative commercial practices (Zuboff, 2019), incapable of imagining different digital futures (Markham, 2021) because they have perhaps internalized this exploitation within hegemonic narratives of dependency, inevitabilityand trajectorism (Markham, 2021).

This research highlights how researchers can play a scholar-as-activist role to elicit critical reflections regarding structures of datafication and surveillance. Drawing on a critical pedagogy approach (Markham, 2019), I prepared a "7-day autoethnography challenge," with many prompts directly inspired by MMS. I invited my own students to participate, aiming to help them become autoethnographers of their own lives, i.e. to stimulate reflections about their practices related to algorithmic media and how their experiences are increasingly intertwined with datafication and surveillance processes, inorder to increase their self-reflexivity, awareness and data literacy. Although this study analyses how algorithmic outputs are embedded in everyday life (Siles et al., 2019)--a slightly different focus from the MMS project--the research exemplifies how an MMS approach can turn personal experience into moments of critical understanding of social life.

\section{Subversion and reflexivity}

During the pandemic, for all the obvious reasons, my (Devina's) doctoral work on the use of TikTok and Instagram by 10-18 year-olds in India stalled. At the same time, I hadstumbled into the MMS project to help me deal with some of the disruptions in my own life and work. As the 21 days of prompts progressed, my participation helped my own sense-making of the micro and macro in the pandemic context but also my research approach. Among my research participants, conversations similar to those at MMS weretaking place --not about the pandemic, necessarily, but about the micro aspects of online learning and macro considerations about where we are headed, why this is important and what is coming next, especially because of thoughts of the "new normal." Similar to what was being addressed in the videos, artworks and writing I was 
seeing in the Facebook group for MMS, my encounters with research participants became a space for reflection and voicing confusions/frustrations even as they were questions without answers/solutions.

Subverting the normative structures, as Taylor (2021) discusses, within which we doresearch or work with participants in studies, is especially difficult for entry level researchers, since these structures seem very dominant. Mentoring is "a complex ecology and expanded pedagogy" (Taylor) and can be a way of either reinforcing patterns of institutional structures or subverting these structures, making space for greater independence and exploration of what interdependence means in practice.

The MMS experience represented this sort of mentorship for me. The facilitators playeda role, but the digital interface of Facebook also became a "mentor" by showing me howresearch collaborations are emergent, sometimes even accidental, and how connections emerge within scholarship across many boundaries.

\section{Conclusion}

MMS problematizes "taken-for-granted" traits of what it means to do research. Our different experiences highlight that the collaborative parts of autoethnography can be a critical pedagogy practice, in that the interactions and processes, played out in MMS, can help individuals explore their own identities and sensemaking processes, while considering and potentially challenging the constraints of the environments in which these processes are situated. The autoethnographic challenge and this mode of inquiry may enhance self-reflexive practices, the construction and reconciliation of identity, and qualitative ways of knowing that can empower individuals to construct embodied sensibilities toward their daily activities and transform individual "micro" experiences intoa critical connection with the more "macro" community.

\section{References}

Alexander, B.K. (2021). Critical Autoethnography as Intersectional Praxis. In R. Boylornand M. Orbe, Critical autoethnography. Routledge.

Markham, A. (2019). Critical pedagogy as a response to datafication. Qualitativelnquiry, 25(8), 754-760. https://doi.org/10.1177/1077800418809470

Markham, A. (2021). The limits of the imaginary. New Media \& Society, 23(2), 382405.https://doi.org/10.1177/1461444820929322

Siles, I., Espinoza-Rojas, J., Naranjo, A., \& Tristán, M. F. (2019). The mutual domestication of users and algorithmic recommendations on Netflix. Communication, Culture \& Critique, 12(4), 499-518. https://doi.org/10.1093/ccc/tcz025 
Taylor, J. (2021) Subversive Spaces, Embodied Places and Mentoring as Onto-Epistemology. In Mentoring and Communication. Peter Lang.

Wenger, E. (1999). Communities of practice. Cambridge University.

Zuboff, S. (2019). The Age of Surveillance Capitalism. Profile Books. 


\title{
SEEING, BELIEVING, LEARNING: TOWARDS A MANIFESTO
}

\author{
M.E. Luka \\ University of Toronto-Scarborough
}

\section{Background}

On October 27, 2020, 40 people from around the English-speaking world met online to screen a collection of 14 videos exhorting social awareness and change. It was the firstnight of a three-day international performance festival, the Mark De Garmo Virtual International Arts (VIA) Festival for Social Change, on the pandemic's ubiquitous gathering platform, zoom. The first night featured videos generated by the 21-day autoethnographic initiative, "Massive and Microscopic Sensemaking (MMS) in times of COVID 19" (Markham, Harris \& Luka 2020) that had ended in June. The presentation honoured the independent and interdependent methods and methodologies finessed throughout the MMS experience, including the messy production values required by therapid response process during the challenge, but also presented unresolved and sometimes inchoate calls to social action engendered by the global COVID-19 pandemic and subsequent social protests. Interspersed with discussions, the hourlongevent created a bubble of visceral, intense reminders about what it had been like in the first weeks of the lockdowns, within which to watch, revisit, feel, and reflect on COVID- 19 times, so far. Months later, it is evident that the layers of observation, reaction and early analysis generated within the MMS experience allowed scholars, artists and activists a way to feel connected to others throughout startling shifts in unfolding social conditions, and to actively pause their own research agendas in pursuit of a digitally vibrant collective research exchange.

In this paper, the first author explores how notions of resilience embedded in the video productions can be unpacked as expressions not just of the tantalizing sensemaking promise of MMS, but also how MMS offers lessons about collective research actions and lays the groundwork for a manifesto to encourage further work in this vein. At the end of the paper, the rest of the authors in this panel will present a co-created Massive Micro Sensemaking manifesto to discuss at AolR during the question and answer period for this panel. To what extent will the shifts in research and creative practice that were wrenched online--or deepened through virtual spaces and methods and the kind ofcollegiality engendered by MMS with its 165 participants--take hold in the longer term, and why does this matter? Peering through the lenses of the videos in the VIA Festival presentation illustrate how the MMS process moved participants towards the making of a manifesto on collaborative, or collective, actions and shared experiences.

\section{Lessons learned: towards a manifesto}

Digital feminist manifestos surface periodically in internet studies and digital media forums, particularly in relation to efforts towards collaboration, addressing power 
injustices and anti-racism, class inequities and more (Kember, 2012; Moravec, 2018). Indeed, as Moravec (2018) notes: "while manifestos are by their nature utopian visions,...the more prosaic definitions also contain words like "hope," "promise," "potential," and "future" (p.189). Such terminology resonates throughout the MMS experience, including the VIA Festival videos. This is more than simply a set of demands for a better world. In the digital context, manifestos are used to articulate social problems and actionable tasks and questions to address them. In critical internet studies, manifestos can help identify research questions, narratives about the problems involved, and how the questions could be addressed. Art and creative production are crucial to this exercise. Hence, Natalie Loveless's manifesto on research-creation as a mode of scholarly production and analysis (2019) argues "... [that] the crafting of a research question is the crafting of a story that is also the crafting of an ethics" (pp. 24- 25, emphasis in the original). To arrive at this position, Loveless examines the affective work required to study inter-species relations in Donna Haraway's (2003) manifesto on "companion species" as both a defamiliarizing and ethical storytelling strategy. This methodological approach identifies circumstances within which the person doing the investigation is deeply implicated, never more so than on the internet through the kind ofcollective research and work undertaken at MMS. In the MMS context, identifying "Haraway's dog" (as multi-faceted objects of study) is similar to asking how to denaturalize the situation, the conditions, the people and other-than-people that intersected at the specific moment of the global pandemic.

But that's not all. The rapid and repetitive responses in the MMS videos speak to a desire for urgent collective research and curiosity about the varied answers to autoethnographic questions in the Covid context, such as "why is this important?," "who cares?," "why?" and "what next?" These questions prompt crucial forms of observation at different registers (visceral, analytical, narrative, descriptive, etc.), evident in the otherpapers in this panel, and are often explicitly grounded in visual or oral culture practices, contributing to the painting of a picture or the telling of a story that can act as a call to action, or a lesson learned.

\section{Resilience, repetition, retelling: collective ethical sensemaking}

In the MMS case, telling the story of the unfolding responses to pandemic social conditions in video form became a way to reflect on what can be understood as steadfast forms of resilience in a social context. The notion of resilience in the spheres of the arts, digital media, social media and internet production is often mobilized to signal strength of will and creative talent; a primarily laudatory and desired characteristicfor workers in the sector, who experience precarity and often damaging work conditions while expected to be inventive and productive (McRobbie 2020). The MMS videos collectively offer a palpable desire to make sense of the world outside the MMS space and time while situated within it: to be resilient and to show resilience at specific sites of storytelling. But many of the MMS videos also hint at unresolvable devastating personal and social experiences during the first half-year of the COVID-19 pandemic. And while these representations do not compare to the difficulties of resisting racism and other systemic oppressions that became increasingly visible in the 
pandemic--including BlackLives Matter protests--the sincere and attentive reworking of the pandemic stories offered lessons to an increasingly skillful research-creation community of practice that might contribute to resolving complex challenges.

So, what next? What can we do about pandemics and social conditions alike as researchers, creators, and activists? Concluding with a co-created manifesto by panel participants, this final paper challenges the internet research community to consider how large-scale collaborations continue to have strong reach and duration through care-full nurturing and digital curating.

\section{References}

Haraway, D. (2003). The Companion Species Manifesto. Prickly Paradigm Press.

Kember, S. (2012). "Notes Towards a Feminist Futurist Manifesto," Ada: A Journal ofGender New Media and Technology 1.

Loveless, N. (2019). How to Make Art at the End of the World: A Manifesto forResearch-Creation. Duke University Press.

Markham, A., Harris, A., \& Luka, M. E. (2020). Massive and microscopic sensemakingin times of COVID-19. Qualitative Inquiry, $10.1177 / 1077800420962477$

McRobbie, A. (2020). Feminism and the Politics of Resilience. In Feminism and the Politics of Resilience: Essays on Gender, Media \& the End of Welfare, pp. 42-72. Polity.

Moravec, M. (2018). Exceptionalism in Digital Humanities: Community, Collaboration \&Consensus. In Disrupting the Digital Humanities, eds., D. Kim \& J. Stommel, pp. 169-196. Punctum Books. 